\title{
Risk of second primary cancers among patients with a first primary gastric cancer: A population-based study in North Portugal
}

\author{
Samantha Morais ${ }^{\mathrm{a}}$, Luís Antunes ${ }^{\mathrm{b}}$, Maria José Bento ${ }^{\mathrm{b}}$, Nuno Lunet ${ }^{\mathrm{a}, \mathrm{c}, *}$ \\ a EPIUnit - Instituto de Saúde Pública, Universidade do Porto, Rua das Taipas, no 135, 4050-600 Porto, Portugal \\ b Registo Oncológico Regional do Norte (RORENO) - Instituto Português de Oncologia do Porto, Rua Dr. António Bernardino de Almeida, $4200-072$ Porto, Portugal

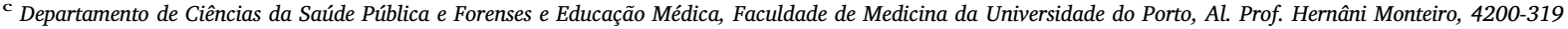 \\ Porto, Portugal
}

\section{A R T I C L E I N F O}

\section{Keywords:}

Epidemiology

Gastric neoplasms

Population register

Second primary neoplasm

\begin{abstract}
A B S T R A C T
Background: The growing number of incident cases of gastric cancer along with improved survival result in a rising population of survivors at risk of second primary cancers (SPC). We estimated the cumulative incidence of metachronous (diagnosed $>2$ months after first primary cancer [FPC]) SPC in gastric FPC patients and compared the incidence of metachronous SPC with that expected in the general population.

Methods: A cohort of gastric FPC patients from the North Region Cancer Registry of Portugal, diagnosed in 2000-2006 ( $\mathrm{n}=7427$ ) was followed to 31 December 2010 for synchronous and metachronous SPCs. Cumulative incidence of metachronous SPCs taking into account death as a competing event and standardized incidence ratios (SIR) of metachronous SPCs were estimated.

Results: Overall, 331 (4.5\%) patients developed an SPC (26.9\% synchronous and $73.1 \%$ metachronous). Over half of the SPCs occurred in digestive organs. Among men, the most frequent were colon, prostate, and trachea, bronchus and lung; in women, colon, breast and thyroid were the most common. The 10-year cumulative incidence of metachronous SPC for males was $5.7 \%$ and for females 3.5\%. The SIR for all cancers was 1.30 in males and 1.20 in females. Among both sexes, significantly higher SIRs were observed for cancers of the oesophagus (males: 4.99; females: 8.03), small intestine (males: 11.04; females: 13.09) and colon (males: 2.42; females: 2.58).

Conclusions: Patients with a gastric FPC were found to be at increased risk of developing SPC, mainly in digestive organs, when compared to the general population. Close surveillance of these patients may allow early detection of SPC.
\end{abstract}

\section{Introduction}

Gastric cancer is the fifth most common and third leading cause of cancer death worldwide [1]. Downward trends in mortality have been observed, however, the declines have become gradually smaller in some countries and a levelling off may be expected in high-income settings [2]. In Portugal, gastric cancer ranks fifth in incidence and mortality [1]. Despite the latter showing a decreasing trend in the past few decades [3], Portugal continues to present the highest mortality rates in Western Europe [4] and there is a large variation within the country; in the North region, incidence and mortality are much higher $[3,5]$.

Though survival from gastric cancer remains poor [6], an increase has been observed due to gradual improvements in diagnosis and treatment [7]. These survivors are at increased risk for several adverse health events, including recurrence of first primary cancer (FPC), cardiovascular diseases or second primary cancer (SPC) [8]. Between 1995 and 1999, the stomach was the fifth most frequent FPC site of multiple tumours, accounting for $4.1 \%$ of all subsequent cancers in Europe [9]. In the United States of America (USA), $4.0 \%$ of patients with an initial diagnosis of stomach cancer from 1973 to 2000 and a maximum followup of 27-years developed a subsequent primary cancer [8]. In Northern Portugal, stomach cancer has been shown to be the third most common FPC for patients diagnosed with a subsequent tumour between 2000 and 2003 , contributing to an estimated $9.5 \%$ of all multiple primary cancers [10].

In the present study, we expanded previous observations in the North of Portugal, by following for a maximum of 10-years a population-based cohort of patients with a gastric FPC, diagnosed between

\footnotetext{
* Corresponding author at: Departamento de Ciências da Saúde Pública e Forenses e Educação Médica, Faculdade de Medicina da Universidade do Porto, Al. Prof. Hernâni Monteiro, 4200 - 319 Porto, Portugal.

E-mail address: nlunet@med.up.pt (N. Lunet).
} 
2000 and 2006, for the occurrence of synchronous and metachronous SPCs. Our aim was to estimate the 10-year cumulative incidence of metachronous SPC, taking into account the competing event of death, and to compare the incidence of metachronous SPC in gastric FPC patients with the expected incidence in a sex-, age- and calendar yearmatched population.

\section{Methods}

\subsection{Study setting}

Cancer data were provided by the North Region Cancer Registry (RORENO), a population-based cancer registry established in 1988. The registry covers the Northern region of Portugal, corresponding to approximately 3.3 million inhabitants, which is nearly one-third of the Portuguese population. All incident cancers occurring in the area are recorded by the registry, either directly from the main public hospitals through a web-based platform, or based on hard copies of medical reports from private hospitals and pathology laboratories. RORENO calculates cancer incidence using estimates of the resident population in the area covered by the registry each year, according to Statistics Portugal. The results are expressed as an annual rate per 100000 person-years. Registration follows the International Agency for Research on Cancer (IARC) rules which include four quality dimensions: comparability, validity, timeliness and completeness. Registries maintain quality through regular screening with pre-defined algorithms for validity and consistency [11]. From 1998 to 2002, RORENO fulfilled IARC indices of data quality, which indicates a high degree of completeness of ascertainment $[12,13]$.

\subsection{Tumour classification and definition of multiple primary cancers}

Tumour topography and morphology were classified according to the International Classification of Diseases for Oncology, Third Edition [14], and then recoded to the International Statistical Classification of Diseases and Related Health Problems 10th Revision [15].

SPC is a new primary cancer in a person with a history of malignancy [16]. Multiple primary cancers were defined according to the guidelines proposed by the International Association of Cancer Registries (IACR) and IARC [17]. Briefly, these guidelines consider primary cancers those that originally developed in an organ or tissue, not being an extension, recurrence or metastasis. Different morphologies (even with the same topography) or dissimilar topographies should be regarded as multiple primary cancers, regardless of the time between diagnoses, unless they correspond to systemic cancers, which are considered the same cancer.

\subsection{Study design}

All primary invasive tumours of the stomach (C16) diagnosed in adults resident in the North of Portugal between 1 January 2000 and 31 December 2006 were identified $(\mathrm{n}=8174)$.

We excluded patients who had a diagnosis of an FPC, except skin non melanoma, previous to a stomach cancer $(n=428)$ and those which could not be linked to the National Health System for assessment of vital status $(\mathrm{n}=319)$. The latter were not significantly different from included participants regarding sex (men: $64.3 \%$ vs. $59.3 \%$; $=0.075$ ) but were significantly older at diagnosis of FPC (median: 72 vs. 68; $\mathrm{p}=0.002$ ), and had significantly more gastric not otherwise specified and less non-cardia tumours $(90.6 \%$ vs. $66.3 \%$ and $5.6 \%$ vs. $27.9 \%$, respectively; $\mathrm{p}<0.001$ ).

The remaining patients $(n=7427)$ were followed to 31 December 2010, allowing for over 10-years of potential follow-up, until the diagnosis of an SPC or death, whichever occurred first. The occurrence of any subsequent cancer was ascertained by means of record linkage with the list of cases registered by RORENO. Patients known to have died but with an unknown date of death $(n=18)$, were imputed a follow-up time equal to the median follow-up of the corresponding sex, 5-year age group (from 15-19 to 70-74, and $\geq 75$ ) and year of diagnosis.

Whenever more than two primary cancers were observed in the same patient, only the first was considered; third and subsequent primaries were disregarded for the present analysis. Due to the thorough evaluation of cancer patients during the initial medical work-up [8], we classified SPCs as synchronous when diagnosed within two months of the gastric FPC or metachronous otherwise, as previous studies on this topic $[8,10,18,19]$.

\subsection{Statistical analysis}

Patients' characteristics were presented as counts and proportions for categorical variables, and median (percentile 25-percentile 75 [P25P75]) for quantitative variables. To compare quantitative and categorical variables across groups, the Mann-Whitney test and Chi-square test were used, respectively. Statistical significance was considered when $\mathrm{p}<0.05$. All reported $p$-values are two-sided. These analyses were carried out separately for synchronous and metachronous SPCs.

Cumulative incidence and corresponding 95\% confidence intervals (CI), stratified by sex, age and tumour location, for the occurrence of metachronous SPC were calculated, with death as a competing event according to the method introduced by Kalbfleisch and Prentice [20]. Briefly, this method allows for patients who died to no longer be at risk for an SPC by actively removing individuals from the risk sets; thus, it differs from the cumulative incidence estimated by the Kaplan-Meier (KM) method, which treats competing events as censored at the time they occurred. We show plots for metachronous SPCs and mortality [21].

Standardized incidence ratios (SIR) and corresponding 95\%CI were computed to compare cancer incidence rates among gastric FPC with those in the general population. SIRs were calculated by dividing the observed number of metachronous SPCs by the expected number of cases, in the same calendar year, if the cancer incidence rates in the general population had been observed among survivors. The latter were estimated by multiplying the cancer incidence in the general population by the person-years at risk (PYAR) in the corresponding stratum; defined according to sex, 5-year age group (from 15-19 to 70-74, and $\geq 75$ years) at FPC diagnosis and each individual calendar year (2000-2010). PYAR were calculated from two months after the diagnosis of FPC until diagnosis of a metachronous SPC, death or end of follow-up (31 December 2010), whichever occurred first. The corresponding stratum incidence of cancer among the general Northern Portuguese population was acquired from RORENO (described in 2.1 Study setting) [22]. The 95\%CIs were estimated assuming that the observed number of cancers followed a Poisson probability distribution.

In addition, several sensitivity analyses were performed defining metachronous SPCs as diagnosed one month, six months and one year after the gastric FPC.

Statistical analyses were conducted using STATA $^{\circ} 11.2$ and R3.3.2 (cmprsk package). The study was approved by the Ethics Committee of the Portuguese Institute of Oncology of Porto (Ref. CES IPO: 173/2015) and the analyses were performed according to RORENO guidelines ensuring the anonymity of information used.

\section{Results}

A total of 7427 cases of gastric FPC diagnosed between 2000 and 2006 were included. During follow-up, we found 331 (4.5\%) SPCs, 242 (73.1\%) were classified as metachronous (Fig. 1). When the time to define SPCs was changed, the number of metachronous SPCs decreased from $264(79.8 \%)$ to 185 (55.9\%) using one month and one year, respectively (Appendix A).

Patientś characteristics are shown in Table 1. The median age at diagnosis of FPC did not differ between patients with and without an 
From 1 January 2000 to 31 December 2006

8174 patients with newly diagnosed gastric cancer
Fig. 1. Flow chart of patient inclusion in the study (Follow-up to 31 December 2010).

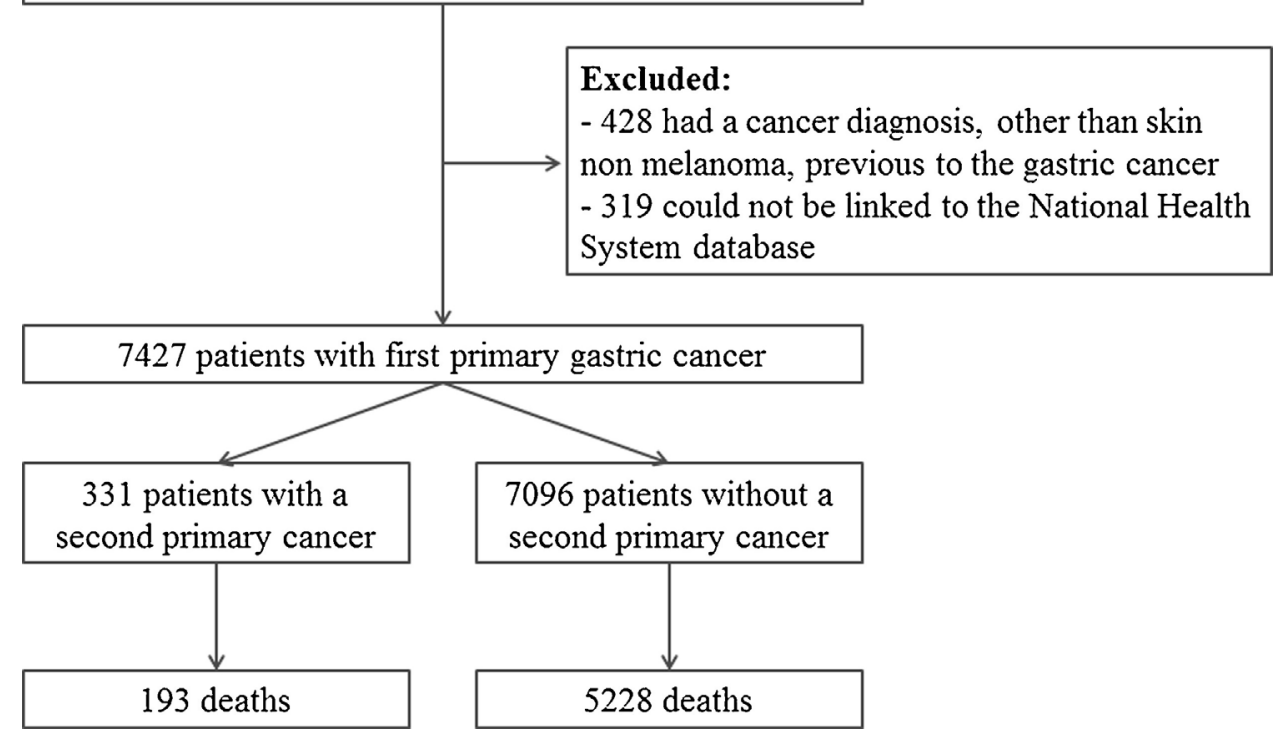

SPC. More than half $(59.3 \%)$ of the patients were male, with SPCs occurring more often in men. The distribution of tumour location within the stomach was similar between patients with a synchronous or metachronous SPC and without an SPC. The median (P25-P75) follow-up in patients without an SPC was 7.3 (5.5-8.9) years, with a synchronous SPC was $0.1(0.0-0.1)$ years, and $2.4(1.0-4.7)$ years for patients with a metachronous SPC.

The SPC distribution showed that the most common sites were digestive organs $(n=169,51.1 \%$ ), over two-thirds were classified as metachronous tumours. In men, the most frequent synchronous SPC were the colon $(n=24,42.9 \%)$, rectum $(n=5,8.9 \%)$, and trachea, bronchus and lung $(\mathrm{n}=5,8.9 \%)$; for metachronous SPC, the colon $(\mathrm{n}=35,20.8 \%)$, prostate $(\mathrm{n}=33,19.6 \%)$, and trachea, bronchus and lung ( $\mathrm{n}=22,13.1 \%$ ) were the most frequent. Among women, the colon $(\mathrm{n}=11,33.3 \%)$, breast $(\mathrm{n}=4,12.1 \%)$ and non-Hodgkin lymphoma ( $\mathrm{n}=3,9.1 \%$ ) were the most common synchronous SPC. Colon $(n=20,27.4 \%)$ and breast $(n=15,20.5 \%)$ cancers occurred frequently as metachronous SPC in women (Fig. 2). The most frequent metachronous SPCs among both sexes remained the same regardless of the time definition used (Appendix A). Among males, over $80 \%$ of SPCs in the prostate, bladder, and liver and intrahepatic bile ducts were metachronous tumours diagnosed more than six months after gastric FPC diagnosis. In females, all rectum SPCs were synchronous tumours diagnosed within one month of the gastric FPC; while all SPCs of the cervix uteri, kidney, and trachea, bronchus and lung occurred over six months following the gastric FPC.

The cumulative incidence of metachronous SPCs was calculated with death as a competing event (Fig. 3). The overall 10-year cumulative incidence of SPC was $4.8 \%$ (males: $5.7 \%$; females: $3.5 \%$ ); the overall 10-year cumulative mortality was $69.5 \%$ (males: $72.0 \%$;

Table 1

Features of gastric cancer patients with synchronous and metachronous second primary cancers, and without a second primary cancer.

\begin{tabular}{|c|c|c|c|c|c|c|c|c|}
\hline \multirow[b]{2}{*}{$\mathrm{N}$} & \multirow{2}{*}{$\begin{array}{c}\text { Total } \\
\mathrm{N}=7427 \\
(100.0 \%)\end{array}$} & \multicolumn{2}{|c|}{$\begin{array}{l}\text { Patients without an SPC } \\
\qquad N=7096(95.5 \%)\end{array}$} & \multicolumn{5}{|c|}{$\begin{array}{c}\text { Patients with an SPC } \\
\quad \mathrm{N}=331(4.5 \%)\end{array}$} \\
\hline & & & $\begin{array}{l}\text { p-value } \\
\text { All SPC vs. } \\
\text { no SPC }\end{array}$ & $\begin{array}{l}\text { Synchronous } \\
89\end{array}$ & $\begin{array}{c}\text { p-value } \\
\text { SynchronousSPC } \\
\text { vs. no SPC }\end{array}$ & $\begin{array}{l}\text { Metachronous } \\
242\end{array}$ & $\begin{array}{c}\text { p-value } \\
\text { Metachronous SPC } \\
\text { vs. no SPC }^{\text {a }}\end{array}$ & $\begin{array}{c}\text { p-value } \\
\text { Synchronous SPC } \\
\text { vs. } \\
\text { Metachronous SPC }\end{array}$ \\
\hline $\begin{array}{l}\text { Age at diagnosis of GC, } \\
\quad \text { years } \\
\text { (median }[\mathrm{P} 25-\mathrm{P} 75]) \\
\text { Sex }(\mathrm{N}[\%])\end{array}$ & $68.0(56.0-76.0)$ & $\begin{array}{c}68.0 \\
(56.0-76.0)\end{array}$ & 0.206 & $69.0(57.0-77.0)$ & 0.843 & $67.0(58.0-73.0)$ & 0.106 & 0.236 \\
\hline Males & $4402(59.3)$ & $4178(58.9)$ & & $56(62.9)$ & & $168(69.4)$ & & \\
\hline Females & 3025 (40.7) & $2918(41.1)$ & 0.001 & $33(37.1)$ & 0.441 & $74(30.6)$ & 0.001 & 0.262 \\
\hline \multicolumn{9}{|c|}{ Tumour location $(\mathrm{N}[\%])^{b}$} \\
\hline Cardia & $436(5.9)$ & $419(5.9)$ & & $3(3.4)$ & & $14(5.8)$ & & \\
\hline Non-cardia & 2070 (27.9) & $1983(28.0)$ & & $26(29.2)$ & & $61(25.2)$ & & \\
\hline Gastric NOS & $4921(66.3)$ & $4694(66.1)$ & 0.633 & $60(67.4)$ & 0.597 & $167(69.0)$ & 0.629 & 0.56 \\
\hline $\begin{array}{l}\text { Follow-up, years } \\
(\text { median }[\text { P25-P75]) }\end{array}$ & $7.0(5.2-8.8)$ & $7.3(5.5-8.9)$ & $<0.001$ & $0.1(0.0-0.1)$ & $<0.001$ & $2.4(1.0-4.7)$ & $<0.001$ & $<0.001$ \\
\hline Dead $(N[\%])^{d}$ & $5426(73.1)$ & $5233(73.8)$ & $<0.001$ & $61(68.5)$ & 0.268 & $132(54.6)$ & $<0.001$ & 0.022 \\
\hline
\end{tabular}

GC gastric cancer, NOS not otherwise specified, P25 percentile 25, P75 percentile 75, SPC second primary cancer.

${ }^{a}$ Patients without an SPC are those who survived more than two months after diagnosis of the FPC.

b Defined as cardia (C16.0), non-cardia (fundus, C16.1; body, C16.2; pyloric, C16.3; pylorus, C16.4; lesser and greater curvature, C16.5-6) and other parts (C16.8-9) [15].

${ }^{\mathrm{c}}$ Follow-up to 31 December 2010 until SPC, death or end of follow-up. Median follow-up time was estimated using the reverse Kaplan-Meier [45].

d Follow-up to 31 December 2010. 

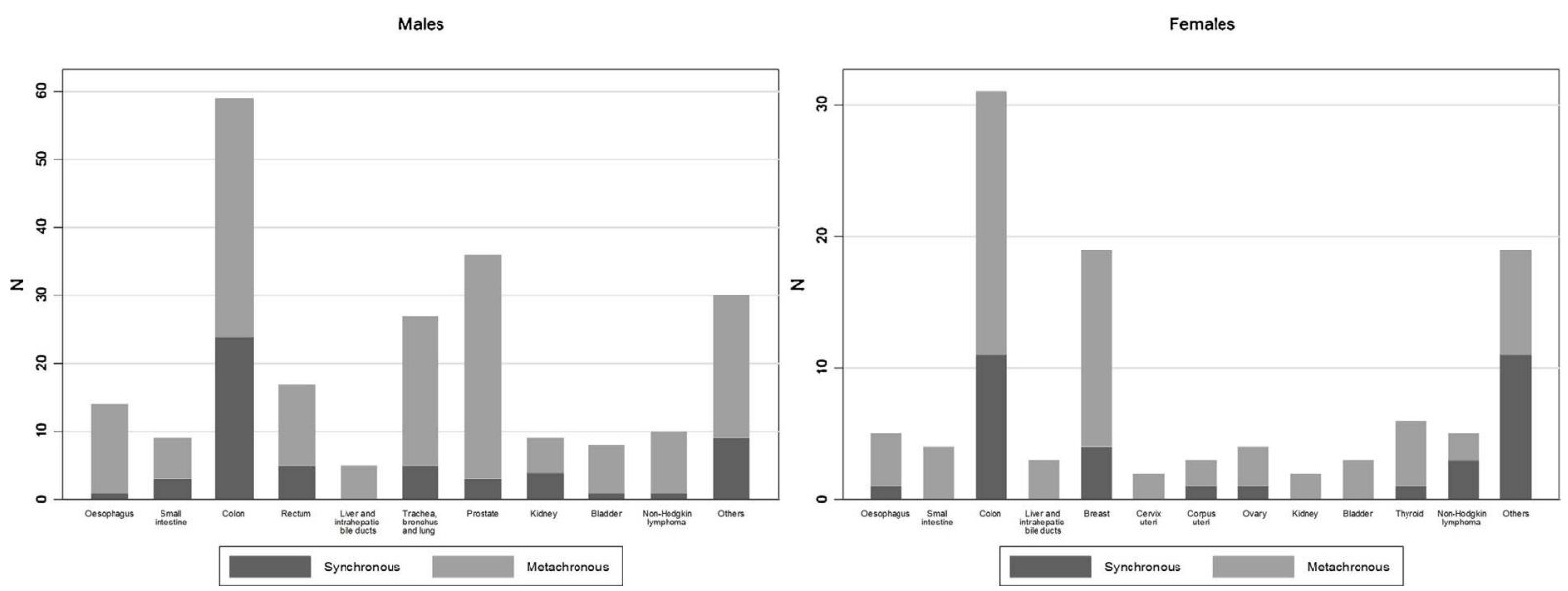

Fig. 2. Synchronous and metachronous second primary cancers ${ }^{\mathrm{a}}$ in males and females.

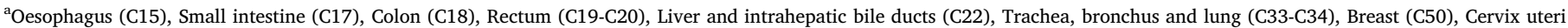

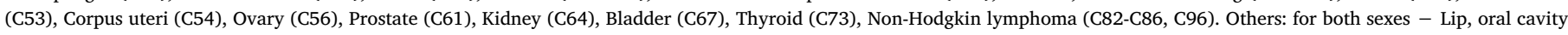

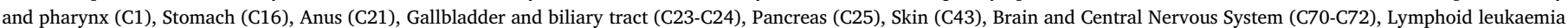

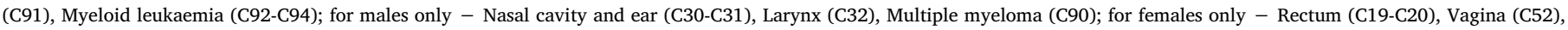
Other and unspecified female genital organs (C57), Renal pelvis (C65), Adrenal gland (C74) [14].

females: $66.0 \%)$. Cumulative incidence estimates were significantly higher among males $(\mathrm{p}<0.001)$ and increased with age ( $\mathrm{p}$ for trend $=0.065$ and 0.556 for males and females, respectively); however, lower estimates were observed in the oldest age group. Among men, non-cardia tumours showed the highest cumulative incidence and in women, not otherwise specified tumours had the highest estimates (Appendix B). Cumulative incidence estimates of metachronous SPCs at 1- and 3-years were higher when using the one month definition and the highest at 10-years for one year metachronous SPCs in both males and females (7.5 and 4.2\%, respectively) (Appendix A). Cumulative mortality was significantly higher among men ( $p<0.001$ ) (Fig. 3) though greater values were observed for older ages and cardia tumours in both sexes (Appendix B).

SIRs $(95 \% \mathrm{CI})$ for the most common metachronous SPCs are shown in Fig. 4. In comparison with the general population, both male (1.30 [1.11-1.52]) and female (1.20 [0.94-1.51]) gastric FPC patients had an increased risk of any type of cancer. Among both sexes, significantly higher SIRs (95\%CI) were observed for cancers of the oesophagus (males: 4.99 [2.66-8.54]; females: 8.03 [2.19-20.56]), small intestine (males: 11.04 [4.05-24.03]; females: 13.08 [3.57-33.52]) and colon (males: 2.42 [1.69-3.37]; females: 2.58 [1.58-3.98]). Males were also at increased risk for non-Hodgkin lymphoma (SIR [95\% CI] 2.53

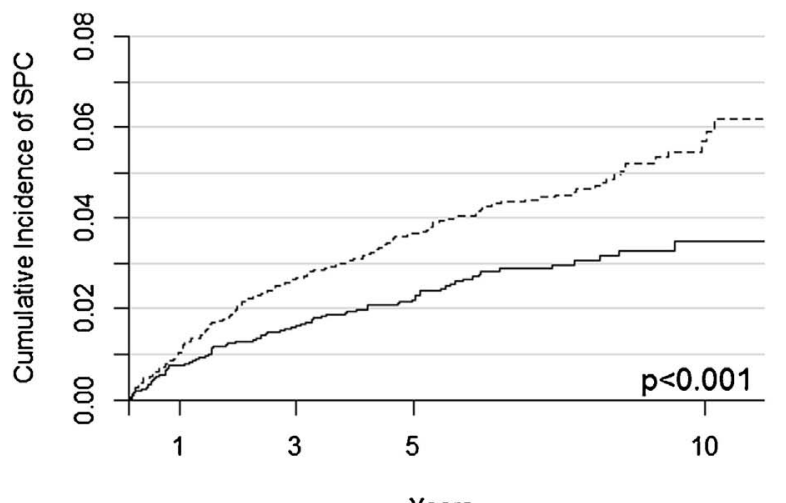

Years
[1.16-4.81]), and females cancer of the liver and intrahepatic bile ducts (SIR [95\% CI] 5.18 [1.07-15.15]). The incidence rate of any metachronous SPC among males was higher than in the general population, though the strength of the association decreased as the cut-off to define metachronous SPCs increased, and significantly higher SIRs were observed for cancers of the oesophagus, small intestine and colon across all four time definitions. For women, the incidence rate of any metachronous SPC was only significantly higher than in the general population when metachronous SPCs were defined using the one-month cutoff, yet as observed in men, the strength of the association declined as the time used to define metachronous SPCs increased; significantly higher SIRs were observed for cancers of the small intestine and colon using all time definitions (Appendix A).

\section{Discussion}

The overall 10-year cumulative incidence of metachronous SPCs and mortality were 4.8 and $69.5 \%$, respectively. Compared to the general population, gastric FPC patients had a significantly increased risk of metachronous SPCs, especially in other digestive organs, which remained even when defining these SPCs as more than one year post diagnosis of the FPC.

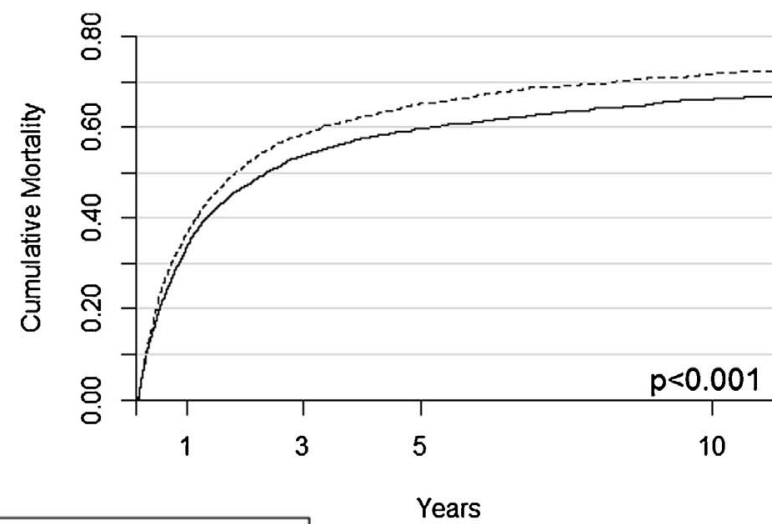

Years

Fig. 3. Cumulative incidence of metachronous second primary cancers, by sex, taking into account the competing event of death. SPC second primary cancer.

*Note that a different scale is used for the two outcomes. 
Males

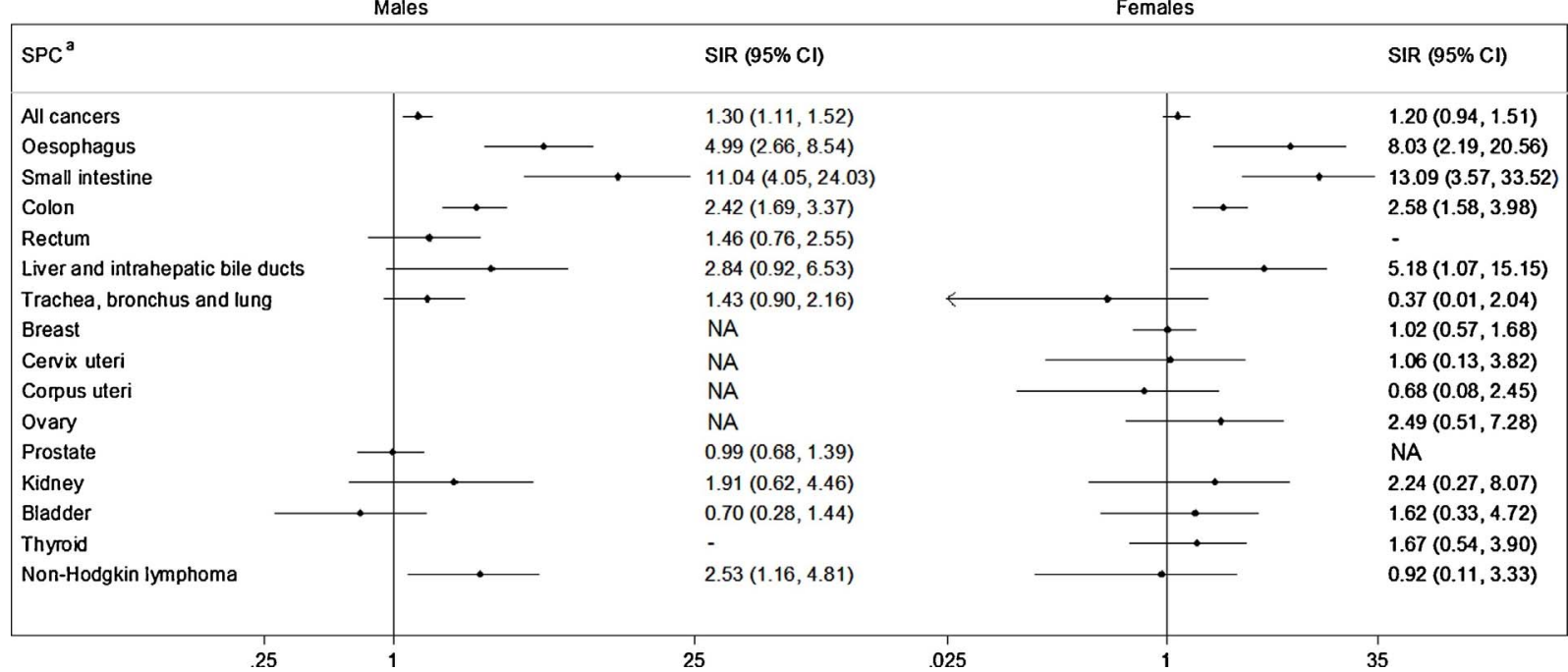

Fig. 4. Standardized incidence ratios and $95 \%$ confidence interval of selected metachronous second primary cancers in patients with first primary gastric cancer. 95\% CI 95\% confidence interval, NA not applicable, SIR standardized incidence ratios; SPC second primary cancer.

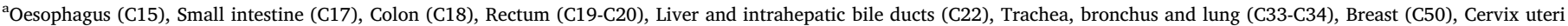
(C53), Corpus uteri (C54), Ovary (C56), Prostate (C61), Kidney (C64), Bladder (C67), Thyroid (C73), Non-Hodgkin lymphoma (C82-C86, C96) [14].

Although some national studies analysing SPCs in gastric cancer patients have been conducted in Sweden [23], Taiwan [24] and the USA [25], comparisons are difficult because of the varying diagnostic and follow-up times, and most other studies are from single-institutions, often with few SPCs. The proportion of SPCs diagnosed in patients with gastric FPC in Northern Portugal was higher than the observed in Sweden, where 962 (2.8\%) SPCs were reported in 34506 people with gastric cancer diagnosed from 1960 to 1981 and followed to December 31, 1981 [23], a longer period than that of our study. On the other hand, our results are consistent with those from Taiwan, where over 2000 SPCs were observed during a follow-up of 47729 gastric FPC patients diagnosed between 1997 and 2011 [24]. In the USA, among 33720 gastric FPC patients diagnosed from January 1992 to December 2012, 1838 patients (5.5\%) developed metachronous SPCs after a period of six months from diagnosis [25]. A previous study also from the North of Portugal, though with a longer study period, had 78 (3.4\%) SPCs (73\% were defined as metachronous using a six month cut-off) diagnosed in 2668 patients with gastric cancer between July 1974 and December 1999 [26]; this overall estimate of SPCs is lower than ours. This may be partially explained by improvements in survival of cancer patients overtime $[6,7]$ and the previous study only had follow-up of patients from a single oncology specialized hospital, while our results are from a population-based cancer registry. In general, the proportion of SPCs that are synchronous varies from 11.4 to $47.9 \%$ [19,24], using a two month and a one year definition, respectively. We found less than one-third synchronous SPCs using a two month definition, while almost half of all SPCs were diagnosed within the first year of the gastric FPC.

The risk of metachronous SPCs after the diagnosis of gastric cancer has been estimated in previous studies: in Korea, patients who underwent curative resection of gastric cancer were retrospectively reviewed showing a 5-year cumulative incidence of SPC of $1.4 \%$ [27], and in Japan, the occurrence of second tumours at least six months following resection of early gastric cancer was estimated at 5.0\% after more than 10 years of follow-up [28]. Another study, from Korea, had an incidence of $3.7 \%$ with a median time to the occurrence of metachronous SPC six months or more after diagnosis of the gastric FPC of 3.3 years (standard deviation: 2.6 years) [29]. Our overall cumulative incidence of metachronous SPCs more than two months after the FPC was 3.1 and $4.8 \%$ at 5- and 10-years, respectively; corresponding estimates using a six month cut-off to define SPCs were 3.3 and 5.4\%, respectively.

The guidelines chosen to diagnose SPC may account for some of the heterogeneity observed; compared to the Surveillance, Epidemiology, and End Results Program coding rules, IARC/IACR rules are less complex, have not changed over time and report fewer multiple primary cancers [30]. The best interval to define SPCs as synchronous or metachronous has also been disputed, ranging from one month to one year $[8,24,31]$. Thus, we may have observed a smaller number of SPCs by using the more restrictive IARC/IACR rules; using a two month gap to classify tumours likely increased the proportion of metachronous SPCs. However, we performed sensitivity analyses using different time periods to classify SPCs as synchronous or metachronous in order to increase comparability with other studies, which use various time definitions.

In published research, the frequency of finding SPC accompanying gastric cancer is higher in male patients $[24,27,28]$, in accordance with our findings. Although SPCs have been related to age [24,28,32], we found no differences between age at diagnosis in patients with and without SPC. Additionally, there were no significant differences in the tumour site of the gastric FPC. Other studies have also not found differences with regards to gastric cancer location [27,28,32].

In our study, the most common types of SPCs were digestive (colon, oesophagus and rectum in males, and colon, oesophagus and small intestine in females). Our data seems to be similar to the findings of others, who also reported that digestive cancers, particularly of the colon and rectum, are the most frequent in gastric cancer patients with multiple malignancies $[23,27,32]$. The high incidence of these cancers in gastric FPC patients may be due to the common carcinogenic process and the same risk factors that affect the gastrointestinal tract (e.g.: contaminants such as $\mathrm{N}$-nitroso compounds found in smoked foods, preserved meats and some alcoholic beverages, diet, and smoking) $[33,34]$. As cancer patients are under closer examination than the general population, the observed increased risks of developing metachronous SPCs in the oesophagus, small intestine and colon after diagnosis of gastric cancer may be due to follow-up of an FPC also in a digestive organ [35]. Additionally, gradual improvements in gastric cancer staging overtime, such as the increasing use of computerised tomography scanning and endoscopic ultrasound [2], may have had some role in the incidence of simultaneous primary cancers. Previous research has also shown significant excess risk for gastrointestinal cancers in patients with a previous gastric cancer diagnosis [24,25]. Accordingly, a prior study in the North of Portugal including patients diagnosed between 2000 and 2003 found that the most common SPC in 
gastric cancer patients were also the colon and rectum [10]; however, it did not show a significant risk of SPCs diagnosed more than two or 12 months after the gastric FPC [36]. In the present study, because of the larger sample size and longer follow-up period, we observed a significant increased risk of SPC after two months in males and though our estimate for SPCs defined using a 12 month gap was not statistically significant, it was more precise. The higher risk of other digestive cancers developing in gastric FPC patients suggests the potential for early detection through careful follow-up of these patients even following the first year of diagnosis.

Among men, the occurrence of oesophageal, bladder, and trachea, bronchus and lung cancers, which are strongly associated with smoking [37], is in accordance with the frequency of those cancers in Northern Portugal [22] and the frequency of tobacco consumption among men in Portugal [38]. Expectedly, among females, the frequency of synchronous and metachronous second breast cancers was high; while in males, metachronous second prostate cancers were common, which is similar to data from RORENO within the same period for the most frequent neoplasm in each sex [22]. Although organized cancer screening programs in Northern Portugal are relatively recent [39], a previous study based on a representative sample of the adult Portuguese population showed a high use of breast, cervical and prostate cancer screening [40]. Additionally, as cancer screening is more frequent among cancer survivors than in the general population [41,42], a high frequency of these cancers as SPCs may be expected. Nevertheless, our results essentially reflect the fact that these cancers have some of the highest incidence rates and no increased incidence was observed in comparison with the general population.

The 10-year cumulative incidence of SPC two months post FPC diagnosis for males was $5.7 \%$ and for females $3.5 \%$. We expect our results, which account for the competing event of death, to be lower than previously estimated using the 1-KM estimator [43]. This is because they would consider death as a non-informative censoring event where censored patients are considered to have the same probability of experiencing the event of interest, SPC, as those who remain in the risk set [44]. Conceptually, this is an unrealistic situation as a patient who is censored due to death will not develop an SPC. Since patients who will never have an SPC are treated as if they could, the 1-KM estimator overestimates the probability of SPC and underestimates the corresponding survival probability [43]. Our results illustrate the need to analyse the occurrence of SPC taking into account the competing event of death since these are mutually exclusive.

The sample was obtained from RORENO, which is representative of Northern Portuguese gastric cancer survivors though it is not representative of all of Portugal as the North has higher incidence rates of gastric cancer compared to the rest of the country [5], thus extrapolating these results to Portugal should be done cautiously. However, our results are consistent with those observed in countries where gastric cancer is frequent (e.g.: Taiwan, Japan and Korea). Our study has certain limitations. It is possible that the number of deaths was underestimated because of loss to follow-up of significantly older patients; however, less than $5 \%$ patients could not be linked thus we do not expect this to significantly change our results. Although we imputed follow-up for some patients who did not have a date of death in the National System Database, we do not believe that this would overestimate PYAR, which could have led to a larger number of expected SPCs. Additionally, although the use of a population-based cancer registry of this magnitude, allowed us to follow over 7000 gastric cancer patients for at least four years and for a maximum of 10 years, we were limited by the information available in the registry. Thus, we were unable to assess cancer outcomes accounting for factors such as family history, lifestyle (diet, alcohol and smoking), comorbidities and treatment in the SIR calculations which have been shown by some studies to be associated with SPCs $[19,24]$. Finally, our study is limited in interpretation as complete data on histotype and stage of gastric tumours are not available from RORENO.
The risk of distinct SPCs among gastric cancer patients is heterogeneous, highlighting the need to consider specific SPCs rather than the overall risk. Though the survival of these patients remains poor [6], we observed that they are at particularly higher risk of being diagnosed again in other digestive organs. Additionally, the interval between the gastric FPC and the diagnosis of an SPC is relevant, with over half of the SPCs being diagnosed more than one year after the FPC. In conclusion, our findings suggest that improvements in clinical practice via close surveillance and counselling may result in earlier diagnosis of specific SPCs and potentially better prognosis leading to reduce the overall burden of cancer among this growing population.

\section{Ethical approval}

The study was approved by the Ethics Committee of the Portuguese Institute of Oncology of Porto (Ref. CES IPO: 173/2015).

\section{Authorship contribution statement}

The author contributions were as follows: SM collected, performed the statistical analysis and interpreted the data, drafted and revised the manuscript. LA and MJB supplied the data, helped in the interpretation of the data and reviewed the manuscript. NL supervised the analysis and interpretation of data, and reviewed the manuscript. SM and NL defined the study hypotheses and designed the investigation. All authors contributed to the discussion of the results. All authors read and approved the final version of the manuscript.

\section{Conflict of interest}

The authors declare that they have no conflict of interest.

\section{Funding}

This study was funded by FEDER (European Regional Development Fund) through the Operational Programme Competitiveness and Internationalization and national funding from the Foundation for Science and Technology - FCT (Portuguese Ministry of Science, Technology and Higher Education) under the Unidade de Investigação em Epidemiologia - Instituto de Saúde Pública da Universidade do Porto (EPIUnit) (POCI-01-0145-FEDER-006862; Ref. UID/DTP/04750/ 2013). An individual PhD grant attributed to SM (SFRH/BD/102585/ 2014) was funded by FCT and the "Programa Operacional Capital Humano" (POCH/FSE).

\section{Appendix A. and B Supplementary data}

Supplementary data associated with this article can be found, in the online version, at http://dx.doi.org/10.1016/j.canep.2017.08.007.

\section{References}

[1] J. Ferlay, I. Soerjomataram, M. Ervik, R. Dikshit, S. Eser, C. Mathers, M. Rebelo, D. Parkin, D. Forman, F. Bray, GLOBOCAN 2012 v1.0, Cancer Incidence and Mortality Worldwide: IARC CancerBase No. 11 [Internet] Lyon, France: International Agency for Research on Cancer, (2013) http://globocan.iarc.fr . (Accessed 17/12/2016)

[2] A. Ferro, B. Peleteiro, M. Malvezzi, C. Bosetti, P. Bertuccio, F. Levi, E. Negri, C. La Vecchia, N. Lunet, Worldwide trends in gastric cancer mortality (1980-2011), with predictions to 2015, and incidence by subtype, Eur. J. Cancer 50 (7) (2014) 1330-1344.

[3] S. Morais, A. Ferro, A. Bastos, C. Castro, N. Lunet, B. Peleteiro, Trends in gastric cancer mortality and in the prevalence of Helicobacter pylori infection in Portugal, Eur. J. Cancer Prev. 25 (4) (2016) 275-281.

[4] B. Peleteiro, M. Severo, C. La Vecchia, N. Lunet, Model-based patterns in stomach cancer mortality worldwide, Eur. J. Cancer Prev. 23 (6) (2014) 524-531.

[5] N. Lunet, [Helicobacter pylori infection in a region with high risk of gastric cancer] in Portuguese, Arquivos de Medicina 25 (6) (2011) 213-217.

[6] L.A. Anderson, A. Tavilla, H. Brenner, S. Luttmann, C. Navarro, A.T. Gavin, B. Holleczek, B.T. Johnston, M.B. Cook, F. Bannon, M. Sant, Survival for 
oesophageal, stomach and small intestine cancers in Europe 1999-2007: results from EUROCARE-5, Eur. J. Cancer 51 (15) (2015) 2144-2157.

[7] F. Lordick, W. Allum, F. Carneiro, E. Mitry, J. Tabernero, P. Tan, E. Van Cutsem, C. van de Velde, A. Cervantes, Unmet needs and challenges in gastric cancer: the way forward, Cancer Treat. Rev. 40 (6) (2014) 692-700.

[8] R. Curtis, D. Freedman, E. Ron, L. Ries, D. Hacker, B. Edwards, M. Tucker, J.J. Fraumeni, New Malignancies Among Cancer Survivors: SEER Cancer Registries, 1973-2000, National Cancer Institute . NIH Publ. No. 05-5302, Bethesda, MD, 2006.

[9] S. Rosso, R. De Angelis, L. Ciccolallo, E. Carrani, I. Soerjomataram, E. Grande, G. Zigon, H. Brenner, Multiple tumours in survival estimates, Eur. J. Cancer 45 (6) (2009) 1080-1094.

[10] L. Pacheco-Figueiredo, L. Antunes, M.J. Bento, N. Lunet, Evaluation of the frequency of and survival from second primary cancers in North Portugal: a population-based study, Eur. J. Cancer Prev. 2 (6) (2013) 599-606.

[11] J. Ferlay, C. Burkhard, S. Whelan, D. Parkin, Check and Conversion Programs for Cancer Registries, IARC, Lyon, 2005.

[12] International Agency for Research on Cancer, Cancer Incidence in Five Continents Vol IX, IARC, Lyon, France, 2007.

[13] C. Castro, M.J. Bento, N. Lunet, P. Campos, Assessing the completeness of cancer registration using suboptimal death certificate information, Eur. J. Cancer Prev. 21 (5) (2012) 478-479.

[14] World Health Organization, International Classification of Diseases for Oncology, third edition, World Health Organization Geneva, 2000.

[15] World Health Organization, International Statistical Classification of Diseases and Related Health Problems 10th Revision, World Health Organization Geneva, 1992.

[16] H. Krueger, D. McLean, D. Williams, Meaning of SPC, Prog. Exp. Tumor Res. 40 (2008) 7-16.

[17] Working Group Report, International rules for multiple primary cancers (ICD-0 third edition), Eur. J. Cancer Prev. 14 (4) (2005) 307-308.

[18] D.R. Youlden, P.D. Baade, The relative risk of second primary cancers in Queensland, Australia: a retrospective cohort study, BMC Cancer 11 (2011) 83.

[19] Y. Kinoshita, H. Tsukuma, W. Ajiki, N. Kinoshita, A. Oshima, M. Hiratsuka, H. Furukawa, The risk for second primaries in gastric cancer patients: adjuvant therapy and habitual smoking and drinking, J. Epidemiol. 10 (5) (2000) 300-304.

[20] J. Kalbfleisch, R. Prentice, The Analysis of Failure Time Data, 2nd ed., John Wiley \& Sons, New York, 2002.

[21] H.T. Kim, Cumulative incidence in competing risks data and competing risks regression analysis, Clin. Cancer Res. 2 (2 Pt 1) (2007) 559-565.

[22] RORENO, Registo Oncológico Regional do Norte 2010, Porto Portugal, (2013).

[23] G. Lundegardh, L.E. Hansson, O. Nyren, H.O. Adami, U.B. Krusemo, The risk of gastrointestinal and other primary malignant diseases following gastric cancer, Acta Oncol. 30 (1) (1991) 1-6.

[24] S.C. Chen, C.J. Liu, Y.W. Hu, C.M. Yeh, L.Y. Hu, Y.P. Wang, Y.P. Hung, C.H. Tzeng, T.J. Chiou, T.J. Chen, C.J. Teng, Second primary malignancy risk among patients with gastric cancer: a nationwide population-based study in Taiwan, Gastric Cancer 19 (2) (2016) 490-497.

[25] B.K. Shah, A. Khanal, Y. Hewett, Second primary malignancies in adults with gastric cancer - a US population-based study, Front. Oncol. 6 (2016) 82

[26] M. Dinis-Ribeiro, H. Lomba-Viana, R. Silva, L. Moreira-Dias, R. Lomba-Viana, Associated primary tumors in patients with gastric cancer, J. Clin. Gastroenterol. 34 (5) (2002) 533-535.

[27] C. Kim, H. Chon, B. Kang, K. Kim, H.C. Jeung, H. Chung, S. Noh, S. Rha, Prediction of metachronous multiple primary cancers following the curative resection of gastric cancer, BMC Cancer 13 (2013) 394.

[28] Y. Ikeda, M. Saku, F. Kishihara, Y. Maehara, Effective follow-up for recurrence or a second primary cancer in patients with early gastric cancer, Br. J. Surg. 92 (2) (2005) 235-239.

[29] J.Y. Kim, W.Y. Jang, M.H. Heo, K.K. Lee, Y.R. Do, K.U. Park, H.S. Song, Y.N. Kim, Metachronous double primary cancer after diagnosis of gastric cancer, Cancer Res. Treat. 44 (3) (2012) 173-178.

[30] H.K. Weir, C.J. Johnson, K.C. Ward, M.P. Coleman, The effect of multiple primary rules on cancer incidence rates and trends, Cancer Causes Control 27 (3) (2016) 377-390.

[31] C. Dong, K. Hemminki, Second primary neoplasms in 633,964 cancer patients in Sweden, 1958-1996, Int. J. Cancer 93 (2) (2001) 155-161.

[32] M. Lawniczak, A. Gawin, H. Jaroszewicz-Heigelmann, W. Rogoza-Mateja, J. Raszeja-Wyszomirska, A. Bialek, K. Karpinska-Kaczmarczyk, T. Starzynska, Synchronous and metachronous neoplasms in gastric cancer patients: a 23-year study, World J. Gastroenterol. 20 (23) (2014) 7480-7487.

[33] L. Mysuru Shivanna, A. Urooj, A review on dietary and non-dietary risk factors associated with gastrointestinal cancer, J. Gastrointest. Cancer 47 (3) (2016) 247-254.

[34] C.C. Abnet, Carcinogenic food contaminants, Cancer Invest. 25 (3) (2007) 189-196.

[35] M.E. Wood, V. Vogel, A. Ng, L. Foxhall, P. Goodwin, L.B. Travis, Second malignant neoplasms: assessment and strategies for risk reduction, J. Clin. Oncol. 30 (30) (2012) 3734-3745.

[36] L. Pacheco-Figueiredo, L. Antunes, M.J. Bento, N. Lunet, Incidence of second primary cancers in North Portugal-a population-based study, J. Cancer Surviv. 10 (1) (2016) 142-152.

[37] S. Gandini, E. Botteri, S. Iodice, M. Boniol, A.B. Lowenfels, P. Maisonneuve, P. Boyle, Tobacco smoking and cancer: a meta-analysis, Int. J. Cancer 122 (1) (2008) 155-164.

[38] H. Carreira, M. Pereira, A. Azevedo, N. Lunet, Trends in the prevalence of smoking in Portugal: a systematic review, BMC Public Health 12 (2012) 958.

[39] Direçãoao-Geral da Saúde, Programa Nacional para as Doenças Oncológicas: Avaliação e monitorização dos rastreios oncológicos organizados de base populacional de Portugal, Relatório 2015, (2016).

[40] A.R. Costa, S. Silva, P. Moura-Ferreira, M. Villaverde-Cabral, O. Santos, I.D. Carmo, H. Barros, N. Lunet, Cancer screening in Portugal: sex differences in prevalence, awareness of organized programmes and perception of benefits and adverse effects, Health Expect. 20 (2) (2017) 211-220.

[41] M. Corkum, J.A. Hayden, G. Kephart, R. Urquhart, C. Schlievert, G. Porter, Screening for new primary cancers in cancer survivors compared to non-cancer controls: a systematic review and meta-analysis, J. Cancer Surviv. 7 (3) (2013) 455-463.

[42] L. Pacheco-Figueiredo, N. Lunet, Health status, use of healthcare, and socio-economic implications of cancer survivorship in Portugal: results from the Fourth National Health Survey, J. Cancer Surviv. 8 (4) (2014) 611-617.

[43] T.A. Gooley, W. Leisenring, J. Crowley, B.E. Storer, Estimation of failure probabilities in the presence of competing risks: new representations of old estimators, Stat. Med. 8 (6) (1999) 695-706.

[44] G.L. Grunkemeier, R. Jin, M.J. Eijkemans, J.J. Takkenberg, Actual and actuaria probabilities of competing risks: apples and lemons, Ann. Thorac. Surg. 83 (5) (2007) 1586-1592.

[45] M. Schemper, T.L. Smith, A note on quantifying follow-up in studies of failure time, Control. Clin. Trials 17 (4) (1996) 343-346. 